Elsevier Editorial System(tm) for Advances in Space Research Manuscript Draft

Manuscript Number: ASR-D-12-00119R2

Title: The effect of geocenter motion on Jason-2 orbits and the mean sea level

Article Type: Special Issue: Altimetry Calibration

Keywords: Jason-2, geocenter motion, GPS, SLR/DORIS, mean sea level error

Corresponding Author: Dr. Stavros Melachroinos, Ph.D

Corresponding Author's Institution: Stinger Ghaffarian Technologies

First Author: Stavros A Melachroinos, PhD

Order of Authors: Stavros A Melachroinos, PhD; Frank G Lemoine, PhD; Nikita P Zelensky, PhD; David D Rowlands, PhD; Scott B Luthcke, PhD; Oleg Bordyugov

Abstract: We compute a series of Jason-2 GPS and SLR/DORIS-based orbits using ITRF2005 and the std0905 standards (Lemoine et al. 2010). Our GPS and SLR/DORIS orbit data sets span a period of 2 years from cycle 3 (July 2008) to cycle 74 (July 2010). We extract the Jason-2 orbit frame translational parameters per cycle by the means of a Helmert transformation between a set of reference orbits and a set of test orbits. We compare the annual terms of these time-series to the annual terms of two different geocenter motion models where biases and trends have been removed. Subsequently, we include the annual terms of the modeled geocenter motion as a degree-1 loading displacement correction to the GPS and SLR/DORIS tracking network of the POD process. Although the annual geocenter motion correction would reflect a stationary signal in time, under ideal conditions, the whole geocenter motion is a non-stationary process that includes secular trends. Our results suggest that our GSFC Jason-2 GPS-based orbits are closely tied to the center of mass (CM) of the Earth consistent with our current force modeling, whereas GSFC's SLR/DORIS-based orbits are tied to the origin of ITRF2005, which is the center of figure (CF) for sub-secular scales. We quantify the GPS and SLR/DORIS orbit centering and how this impacts the orbit radial error over the globe, which is assimilated into mean sea level (MSL) error, from the omission of the annual term of the geocenter correction. We find that for the SLR/DORIS std0905 orbits, currently used by the oceanographic community, only the negligence of the annual term of the geocenter motion correction results in a $4.67 \pm 3.40 \mathrm{~mm}$ error in the Z-component of the orbit frame which creates $1.06 \pm 2.66 \mathrm{~mm}$ of systematic error in the MSL estimates, mainly due to the uneven distribution of the oceans between the North and South hemisphere.

Suggested Reviewers: 


\title{
The effect of geocenter motion on Jason-2 orbits and the mean sea level
}

S A Melachroinos (1,2), F G Lemoine (1), N P Zelensky (1,2), D D Rowlands (1), S B Luthcke (1), O Bordyugov $(1,2)$

(1) Planetary Geodynamics Branch, NASA Goddard Space Flight Center, Greenbelt USA, Frank.G.Lemoine@nasa.gov

(2) SGT-Inc., Greenbelt, Maryland, USA, Smelachroinos@sgt-inc.com, stavros.melachroinos@nasa.gov

\begin{abstract}
We compute a series of Jason-2 GPS and SLR/DORIS-based orbits using ITRF2005 and the std0905 standards (Lemoine et al. 2010). Our GPS and SLR/DORIS orbit data sets span a period of 2 years from cycle 3 (July 2008) to cycle 74 (July 2010). We extract the Jason-2 orbit frame translational parameters per cycle by the means of a Helmert transformation between a set of reference orbits and a set of test orbits. We compare the annual terms of these time-series to the annual terms of two different geocenter motion models where biases and trends have been removed. Subsequently, we include the annual terms of the modeled geocenter motion as a degree-1 loading displacement correction to the GPS and SLR/DORIS tracking network of the POD process. Although the annual geocenter motion correction would reflect a stationary signal in time, under ideal conditions, the whole geocenter motion is a non-stationary process that includes secular trends. Our results suggest that our GSFC Jason-2 GPS-based orbits are closely tied to the center of mass $(\mathrm{CM})$ of the Earth consistent with our current force modeling, whereas GSFC's SLR/DORIS-based orbits are tied to the origin of ITRF2005, which is the center of figure (CF) for sub-secular scales. We quantify the GPS and $S L R / D O R I S$ orbit centering and how this impacts the orbit radial error over the globe, which is assimilated into mean sea level (MSL) error, from the omission of the annual term of the geocenter correction. We find that for the SLR/DORIS std0905 orbits, currently used by the oceanographic community, only the negligence of the annual term of the geocenter motion correction results in a $-4.67 \pm 3.40 \mathrm{~mm}$ error in the Zcomponent of the orbit frame which creates $1.06 \pm 2.66 \mathrm{~mm}$ of systematic error in the MSL estimates, mainly due to the uneven distribution of the oceans between the North and South hemisphere.
\end{abstract}

Key words: Jason-2, geocenter motion, GPS, SLR/DORIS, mean sea level error

\section{Introduction}

The origin of the International Terrestrial Reference System (ITRS) is defined to be the center of mass of the Earth system, including oceans, atmosphere and continental water (McCarthy and Petit 2004). Ideally, the origin of the International Terrestrial Reference Frame (ITRF), realization of the ITRS, to which the Jason orbits are referenced, should coincide with the mean center of mass (CM) of the entire Earth system (Blewitt 2003). Although, the realization of the reference frame, through the geodetic stations, centered 
in the $\mathrm{CM}$, and the separation from physical processes to which the stations are subject, is a coupled problem. For example, according to the principal of the conservation of momentum, the $\mathrm{CM}$ has to be a kinematic fixed point, invariant to terrestrial dynamic processes. However, the redistribution of masses in the Earth system causes geocenter motion and as such seasonal, annual and trend variations between the CM and the center of figure (geometric center of the outer surface of the solid Earth) of the solid Earth to which the actual ITRF is referenced for sub-secular time scales (Dong et al., 2003; Blewitt et al., 2001). Métivier et al. (2010) have found that global ice melting on the Earth can induce long-term displacements of the geocenter particularly along the Z-axis, toward the North Pole. They have calculated that the geocenter velocity can reach $0.7-0.8$ $\mathrm{mm} / \mathrm{yr}$ and is today most probably between 0.3 and $0.8 \mathrm{~mm} / \mathrm{yr}$. As such, for the purpose of accurate geodetic observations, having access to a nearly instantaneous geocenter is extremely important for those missions that can sense geocenter motions to some extent but are not good enough to measure it well independently (Wu et al., 2012). Furthermore, the $\mathrm{CM}$ is directly related to satellite orbital motion and so is the most appropriate choice to model satellite geodetic measurements (Fritsche et al. 2010), such as altimetry.

From the above, and given the required sea level infrastructure stability of $0.1 \mathrm{~mm} / \mathrm{yr}$ (Cazenave et al. 2009), geocenter motion of the $\mathrm{CM}$ with respect to the $\mathrm{CF}$, ideally, should also be included in the process of precise orbit determination (POD), which is based on the site crust-fixed coordinates of GPS, SLR, and DORIS stations. This movement can be thought of as a global degree-1 loading displacement correction to be applied to the crust-fixed coordinates of the tracking network in order to reference them to the CM of the whole Earth (Cerri et al. 2010). Hitherto, the lack of a community consensus on a geocenter model has not allowed the geocenter to be forward modeled as part of the Jason altimetry orbit standards (Cerri et al. 2010, Zelensky et al. 2010). Therefore, our motivation for this investigation arises from the fact that the realization of an orbital frame for altimetry centered in the CM plays a major role in the definition and calculation of the rates of global MSL rise.

There have been a number of approaches for the determination of the geocenter motion models such as: (1) by measuring the translation of a tracking network relative to the center of the geodetic satellite orbits - the "network shift or geometric approach"; (2) by observing the deformation of the solid Earth due to the surface mass load - the "degree-1 deformation approach" (Kang et al. 2009, Lavallee et al. 2006, Blewitt et al. 2001). Dong et al. (2003) suggest the degree-1 deformation approach produces more stable geocenter estimates. Geocenter estimates based on satellite laser ranging (SLR), have been previously reported by Chen et al. (1999). When compared to estimates based on combined atmosphere, ocean and hydrological model outputs, Chen et al. (1999) found general agreement at the annual period but little correlation at the monthly timescale.

Here, we use two most recent versions of geocenter models: (1) The UT/CSR RL04 monthly geocenter time-series from Cheng et al (2010), which is based on the "network approach". Cheng et al (2010) use SLR data from five geodetic satellites (LAGEOS $1 \&$ 2, Starlette, Ajisai and Stella) to estimate a $5 \times 5$ gravity field along with 3 geocenter parameters (Tx,Ty,Tz). (2) The Swenson et al (2008) time-series based on the "degree-1 
deformation approach". The degree-1 terms of this model are estimated from a combination of data from the Gravity Recovery and Climate Experiment (GRACE) satellite mission and the modeled global atmospheric and oceanic effects to the Stokes coefficients.

The 1st question we try to answer in this investigation is which of the produced Jason-2 orbit frames based on the GPS or the $S L R / D O R I S$ data is more closely centered to the $\mathrm{CM}$. For this purpose, we characterize the spurious signals contained in the translational parameters of the GPS and SLR/DORIS Jason-2 orbit origin. Then we concentrate our efforts to the centering of the North/South component. Following this investigation, the suitability of the geocenter motion models and their consistency when used by each geodetic POD approach, GPS or SLR/DORIS, is examined. Then the error in the MSL from the omission of the geocenter motion is evaluated.

\section{Description of the Jason-2 orbit set}

For the purposes of this investigation we compute the Jason2 precise orbits using a dynamic and a reduced-dynamic approach (Bertiger et al. 1994). The current GSFC Jason-2 dynamic and reduced-dynamic orbits have been computed with GEODYN (Pavlis et al. 2009) processing GPS (Table 1), and combined SLR (Pearlman et al. 2002, Urschl et al. 2007) and DORIS (Tavernier et al. 2006, Willis et al. 2010) data using the std0905 standards outlined in Table 1 of Zelensky et al. (2010) and Table 7 of Lemoine et al. (2010). These standards include the GRACE-derived static gravity field EIGENGL04S1 (Lemoine et al. 2007), the GOT4.7 (Goddard Ocean Tide Model) dynamic tide model (update to Ray, 1999), forward modeling of atmospheric mass flux using ECMWF pressure data (Klinker et al. 2000), a GRACE derived time varying gravity model capturing the annual variation (Luthcke et al. 2006), updated ITRF2005 SLR and DORIS station coordinates using LPOD2005 (Ries, 2008, Luceri and Bianco 2007) and DPOD2005 (Willis et al. 2009) and updated GPS station coordinates and orbits using IGS05 (IGSMAIL-5447, Ferland and Bourrassa 2006). The GPS std0905 standards are outlined in Table 1. The GPS constellation orbits are held fixed to the coordinate set generated from a least squares (LSQ) fit to the IGS05 sp3 orbits. The GPS station positions are held fixed to their IGS05 coordinates. The entire GPS antenna phase center and their associated variations map used are compatible with the IGS05 framework. In the GPS dynamic orbit solution ( $g p s d y n$ ) once-per-revolution (OPR) along \& cross-track accelerations parameters are included. For the SLR/DORIS dynamic orbits 1 OPR parameter is estimated every 24-h in a 24-h long arc. For the gpsdyn orbits OPR parameters are estimated every $15-\mathrm{h}$ in a $30-\mathrm{h}$ arc with six hrs of overlap between adjacent arcs (Melachroinos et al. 2011a). Most of the orbit error due to radiation pressure is characterized by an OPR signal (Zelensky et al. 2010). This signal is largely removed upon the estimation of empirical OPR acceleration parameters in the orbit solution (Colombo 1986). However, complex errors in the radiation pressure model interact with the estimated empirical OPR parameters to create errors largely in the $\mathrm{X}$ and $\mathrm{Y}$, and to a smaller extent, to the $\mathrm{Z}$ components of the orbit with a draconitic period of 118-days (Zelensky et al. 2010). Any SRP mis-modeling is expected to have no effect in the annual variation of the $\mathrm{Z}$-component origin. 
Our GPS and SLR/DORIS orbit data sets span a period of 2 years from cycle 3 (July 2008) to cycle 74 (July 2010). Next, we compare our gpsdyn to the SLR/DORIS orbits and the GPS-based reduced-dynamic (gpsred) orbits (Melachroinos et al. 2011b) for internal validation purposes. Also for reasons of external validation we use a set of reduced-dynamic orbits from JPL release-11a standards (jpll 1a) (Bertiger et al. 2010). A reduced-dynamic POD solution is based on the denser and geometrically stronger GPS tracking data (Bertiger et al. 1994, Luthcke et al. 2003). In our gpsred implementation OPR along \& cross-track accelerations are estimated every 30 min with process noise standard deviation of $1.0 \times 10^{-9} \mathrm{~m} / \mathrm{s}^{2}$ and an exponential decay function with a correlation time of $1 \mathrm{hr}$ (Table 1). As demonstrated in Melachroinos et al. (2011a) GSFC's gpsdyn and gpsred orbits agree to within $1 \mathrm{~cm}$ radially with the SLR-DORIS orbits and those computed from other analysis centers (JPL, ESA and CNES), thus satisfying the accuracy requirement of $1 \mathrm{~cm}$ proposed by the oceanographic community (Cazenave et al. 2009). Table 2 summarizes the orbit data sets and their associated acronyms used further in this study.

\section{Spurious signals in the Jason-2 orbit origin}

Based on a GPS LEO tracking approach (Kang et al. 2009) we extract the Jason-2 orbit frame translational parameters per cycle by the means of a Helmert transformation between a set of reference orbits and a set of test orbits. As in Kang et al. (2009), our GPS LEO tracking system consists of the GPS constellation orbits (fixed), the GPS ground station network coordinates (fixed) and the GPS onboard Jason-2 receiver in low Earth orbit. The set of reference orbits is chosen to be the gpsdyn orbits due to the stronger ties to the force modeling. The dynamic technique provides an orbit mostly governed by the dynamic modeling while the reduced dynamic technique provides an orbit mostly tied to the tracking data. The set of test orbits are the jpll1a, SLR/DORIS and gpsred orbits.

To a certain extent, the estimated orbits should follow the TRF origin, as this is defined by the tracking stations, and for the GPS-based orbits, also by the GPS constellation coordinates used. This will greatly depend from the techniques used in the POD processing. In the case of the gpsdyn and SLR/DORIS orbits, the satellite dynamics are constrained by physical models. The transition from satellite states at different measurement times to the state at the solution epoch is furnished by integration of the equations of motion, which are governed mostly by the forces (dynamics) acting on the satellite over the time of interest. As such the gpsdyn and SLR/DORIS orbit should supposedly be closer centered to the CM origin defined by the force modeling used in the POD. In the case of the gpsred orbits, a dynamic and a kinematic tracking technique are combined for the better elimination of errors related to force modeling. Essentially, the gpsred orbits should be closer tied to the ITRF origin defined by the geometry of the denser GPS tracking data. If we suppose that one of the orbits is centered in the CM and the other in the CF, then the estimated translation parameters could represent in reality a geocenter motion based on a geometric approach. An apparent advantage of the satellite tracking approach to interpret the geocenter motion between $\mathrm{CM}$ and $\mathrm{CF}$ is that they 

determine the absolute location of the CM with respect to the Earth's surface (Wu et al. 2012). For the purpose of clarity, and as Collilieux et al. (2009) mention, in the subsecular time scales that we focus on our investigation, we must keep one thing in mind: we are only able to investigate possible translational variations (what we call "geocenter motion") due to the inaccessible constant between CF and CM.

We perform a least squares spectral analysis on the time-series of the estimated translational parameters. As previously stated, a 118-day signal is dominant in the $\mathrm{X}$ and Y components with the largest amplitudes of $2.8 \mathrm{~mm}$ and $2.3 \mathrm{~mm}$ respectively (Fig. 1a and $1 \mathrm{~b}$ ). Especially in the $\mathrm{X}$-component the largest signal comes from the transformation of the gpsdyn and SLR/DORIS dynamic orbits. The 118-day signal is the precise draconitic (beta-prime) period for the Jason satellites and this result supports the earlier discussion about the remaining orbit error due to solar radiation pressure (SRP) mismodeling by Cerri et al. (2010) and Zelensky et al. (2010).

Fig. 1c illustrates that in the Z-component the annual signature has the largest amplitude. Other signals of lower amplitude appear at the 87-days and 112-days period but their origin remains unclear. These signals are very close to the $4^{\text {th }}$ and $3^{\text {rd }}$ harmonics (87-days and 117-days respectively) of the GPS draconitic year of 351 days (Schmid et al. 2007, Ray et al. 2008). The largest annual signature results from the comparison of the gpsdyn to the $S L R / D O R I S$ orbits. The comparison of the gpsdyn to the jpll1a orbits exhibits an annual signature of smaller, but still, of non-negligible amplitude. This demonstrates the presence of residual error in the annual frequency either in the gpsdyn, SLR/DORIS or jpll1a orbits. Does this represent an annual motion between the orbit origins or is it only related to a GPS SRP-induced orbit mis-modelling error, which usually causes orbit variations at the GPS draconitic year of 351 days? For that purpose we use the gpsred orbits since those are less sensitive, by definition, to any dynamic mis-modeling errors (Melachroinos et al. 2011b). The comparison of the Z-component from the gpsdyn to the gpsred orbits does not exhibit any significant signatures (magenta line of Fig. 1c), especially in the annual term but also not in any other draconitic term. The orbit differences in Figures 1a-1c do not show any SRP mis-modeling sensitivity in the Zcomponent, but rather only sensitivity in the $\mathrm{X}$ and $\mathrm{Y}$ components of the std0905 gpsdyn and the SLR/DORIS orbits, at the Jason-2 draconitic period of 118 days. Furthermore, it seems that the gpsred and gpsdyn orbits both have consistent Z-centering. Thus, the 365days signature in the Z-component between the gpsdyn, SLR/DORIS and jpll1a orbits, suggests that it is largely related to an annual motion of the orbit origin. The question that we now need to answer is which of the orbits is more closely centered to the CM?

\section{The north/south centering of the Z-component}

In this section we examine the centering of the orbits and turn our attention to the NorthSouth behavior of the Z-component. As seen previously, the most significant peak of the Jason-2 translational time-series, exhibits an annual signature in the Z-component. We incorporated the 3-dimensionnal annual term from the geocenter motion models of Swenson et al. (2010) and Cheng et al. (2010) inside the Jason-2 POD process (trends 
and biases removed) as a correction to the a-priori position of the tracking stations

$X^{C M}(t)=X^{C F}(t)+X_{C F}^{C M}(t)$

Where

$X^{C F}(t)=X_{0}^{C F}+V_{0}^{C F}(t)+\sum_{i} X_{i}^{C F}(t)$

238 Here $X^{C F}(t)$ is the observed position under a CF frame (ITRF), $X_{0}^{C F}$ and $V_{0}^{C F}$ are position, 239 velocity at reference epoch , defined under the CF frame, $X_{i}^{C F}(t)$ are various nonlinear time-dependent deformations due to solid Earth tide, pole tide, ocean tide, mass loading from atmosphere (not applied here), non-tidal oceans (not applied here), surface groundwater (not applied here), and other local effects, $X_{C F}^{C M}(t)$ in this case is the time-dependent degree-1 loading displacement correction from the annual fitted term of the two geocenter models where any offsets and trends have been removed.

For the Z-component, a shift in the station coordinates from the geocenter correction does not correspond exactly in an equivalent shift in the orbit frame due to the orbit inclination. As such, if there is a shift $d z$ introduced to all a priori coordinates of the ground stations due to the annual term of the geocenter motion correction model, the Zcomponent $T z$ of the orbit will be affected by (Morel and Willis, 2005):

$T z=k \times d z$

Where $k$ is a linear transfer function.

At first, we apply the geocenter motion correction models only in the SLR/DORIS tracking stations of the POD process according to equations (1) and (2). We then compute the Helmert translational parameters between the gpsdyn and the SLR/DORIS orbits. Next, an annual curve to each Z-component time-series is fitted and compared to the annual fitted curves from Swenson et al. (2010) and Cheng et al. (2010). In the comparison we also include the jpl11 $a$ and the gpsred orbits. Fig. 2a illustrates the annual term of the Z-component (black line) by Swenson et al. (2010) and compares it to the Zcomponents of the Helmert transformations between the reference orbit set (gpsdyn) and the test orbits. The SLR/DORIS Z-component annual terms are plotted with and without the annual term of the geocenter motion correction (blue and red). Fig. 2b compares only the annual terms of the SLR/DORIS Z-component to the annual term by Cheng et al. (2010). The Z-component annual amplitudes from the two models and each set of the Jason-2 SLR/DORIS Helmert transformations are summarized in Table 3.

273

From the comparison of the SLR/DORIS orbits without the geocenter motion correction to the gpsdyn orbits (blue line in Fig. 2a and 2b), we find that the amplitude of the Zcomponent annual signature is $2.82 \mathrm{~mm}$. After the introduction of the annual geocenter motion correction from Swenson et al. (2010) and Cheng et al. (2010) in the POD process 
of the SLR/DORIS orbits, the translational variations of the origin drop down in amplitude by $2.10 \mathrm{~mm}$ and $1.17 \mathrm{~mm}$ (red line in Fig. 2a and 2b) respectively. The reduction from the initial annual signature of $2.82 \mathrm{~mm}$ between the SLR/DORIS orbits and the gpsdyn orbits (Table 3), resulted by both geocenter models, represents $25 \%$ of the Swenson et al. (2010) and $58 \%$ of the Cheng et al. (2010) annual term. In this sense the Cheng et al. (2010) model performs the best in reducing the SLR/DORIS-gpsdyn origin translational variations. The total signal reduced with respect to the modeled annual geocenter motion correction is $39 \%$ for both cases. As expected, both models propagate consistently as a correction in the POD process. These results suggest that the $S L R / D O R I S$ orbits are not centered in the CM, whereas, the gpsdyn orbits closely follow the CM origin consistent with the conservative force modeling as this is realized through the GPS POD processing of Jason-2. Furthermore, the 7-parameter transformation between the gpsred and gpsdyn orbits, demonstrates that both orbits sets have a very consistent Z-origin (magenta line in Fig. 2a). Someone would expect that the gpsred orbits would follow the CF as this is defined by the geometry of the denser GPS tracking data, which dominate the reduced dynamic technique. On the contrary the gpsred orbits do not demonstrate any significant Z-origin motion with respect to the gpsdyn orbits, which further supports the argument that both orbits are centered closer to the CM. The annual signature of the transformation between jpll la and GSFC's gpsdyn orbits, (green line in Fig. 2a) exhibits amplitude of $1.66 \mathrm{~mm}$. In section 3, we have shown the observed SRP error does not contribute to the annual signature between the jpll1a and gpsdyn. This fact leaves an open question for further investigation to whether the annual signal seen in the Z-component of $1.66 \mathrm{~mm}$ between the two Jason-2 GPS orbit sets from two different analysis centers is due to an inconsistency in the conservative force modeling of the two solutions (time-variable gravity field) or whether is due to an origin motion.

\section{Geocenter motion and mean sea level}

We have found that the orbit set more closely centered to the CM is the Jason-2 gpsdyn and gpsred orbits. Thus we have succeeded in answering the question opened in section 3. Despite their dynamic definition, the $S L R / D O R I S$ orbits are centered in the CF defined by the $S L R / D O R I S$ network. It's worth noticing that the SLR/DORIS std0905 orbits used in this study is the official product currently released and used by the community for MSL estimations. As such, in this section we will characterize the errors in the MSL studies that would not be seen by the users of satellite altimetry data, when using GSFC's gpsdyn or SLR/DORIS orbits (based on the std0905 standards), without a priori knowledge of the annual geocenter motion.

Primarily, we must be aware that errors in the southern hemisphere MSL estimates (which are differences between the satellite altitude, as this is provided by the estimated orbits, and the radar altimetry data) will have a larger effect due to their statistical overrepresentation in the radar observations (Morel and Willis 2005). As such, we expect that the errors due to the omitted annual geocenter motion over the southern oceans, will have a greater weight. 
In order to characterize these errors and their propagation over the oceans, we perform the Helmert tranformation between the orbits of the same technique, GPS or SLR/DORIS, where in one of the solutions the annual term of the geocenter motion has been applied in the POD process.

The amplitudes of the propagated signals in the Z-component are illustrated in Table 4. The corrected for the geocenter motion SLR/DORIS orbits exhibit a noticeable annual effect in the Z-component of $74 \%$ and $81 \%$ compared to each geocenter model. In the gpsdyn orbits the annual geocenter correction propagates with a ratio of $16 \%$ and $19 \%$ with respect to each model (Table 4). The resulted amplitudes are small. As the transfer function of the origin error is different depending on the technique and processing scheme, the difference between orbits will include a part, which is proportional to the real geocenter motion. In the case of the gpsdyn orbits the resulted ratios are very small.

Looking at the geographical distribution of the amplitudes of the radial orbit differences in Fig. 3, we can note the asymmetry in the North-South direction over water when a land mask is included. The amplitudes of the radial orbit error are significantly larger in the case of the SLR/DORIS orbits and can reach $2.5 \mathrm{~mm}$ in high latitudes depending from the model. The gpsdyn orbits suffer the least from the omission of the geocenter correction. As the transfer function of the origin error is different depending on the technique and processing scheme, the difference between orbits may include a part that is proportional to the real geocenter motion. In the case of the gpsdyn orbits where the geocenter correction has been applied, the resulted ratios are small. The above argument supports the fact that the gpsdyn orbits are indeed centered closer to the CM. Those remain practically insensitive to the geocenter motion correction introduced as a degree-1 loading displacement correction to the tracking stations. Which shows that the real geocenter motion left in the GPS Jason-2 orbits is small since the transfer function resulted after the transformation is also small (see Table 4).

Fig. 4 illustrates the phases of the geocenter motion correction as this propagates over the globe into the orbit's radial component. Even though it is small, it worth noticing that the GPS technique provides a geographical representation of the propagation of the geocenter motion into the radial component, similar to the one from Blewitt et al. (2001).

Fig.5 represents the geographical distribution of the POD omission error on the MSL (in $\mathrm{mm}$ ) resulting from the geocenter motion model of Cheng et al. (2010) in the SLR/DORIS stations for cycle 058 (Jan 28-Feb 07, 2010). The systematic error from the modeled geocenter motion in the Jason-2 SLR/DORIS orbit frame results in a mean Z-component of $-4.67 \pm 3.40 \mathrm{~mm}$. This affects the MSL $(\mathrm{DH})$ by $1.06 \pm 2.66 \mathrm{~mm}$ (Table 5). The systematic error in the Jason-2 gpsdyn orbit frame results in a mean Z-component of only $-0.83 \pm 0.28 \mathrm{~mm}$ which affects the MSL by $0.17 \pm 0.37 \mathrm{~mm}$. We calculated for both Jason-2 gpsdyn and SLR/DORIS orbits and compared to previous studies, the functions (ratios DH/Tz in Table 5) that would result from the Tz error in the orbit frame (and not in the station's TRF), in an error in the MSL, due to the negligence of the geocenter motion. In the case of Beckley et al. (2007), by taking into account $\mathrm{k}=0.74$ from equation (4) of Morel and Willis (2005), the real Tz error in the TOPEX orbit frame 
results into an orbit drift of $1.33 \mathrm{~mm} / \mathrm{yr}$ resulting from a TRF drift of $1.8 \mathrm{~mm} / \mathrm{yr}$ in the stations due to the transition to ITRF2005 from ITRF2000 (Altamimi et al. 2007). This propagates in a $-0.26 \pm 0.72 \mathrm{~mm} / \mathrm{yr}$ error in the MSL, which provides a transfer function of -0.20 , following our approach. In Morel and Willis (2005) a Z shift of $10 \mathrm{~mm}$ in the TRF stations is found to propagate linearly in the orbit frame Z-component by $74 \%$ (= $0.74 \mathrm{~mm} / \mathrm{mm}$ ). Given our approach, the error in the Z-component of the orbit frame resulting from the $10 \mathrm{~mm}$ shift would then be $7.4 \mathrm{~mm}$, which results in an error of 1.21 $\mathrm{mm}$ in the MSL and a transfer function of -0.16 . Our transfer function is closer to Beckley et al. (2007) and differs by 0.05 only from the transfer function of Morel and Willis (2005). It seems that all three results are consistent. We should point out that it is the first time that the study of Morel and Willis (2005) has been verified by real case scenarios. The tiny differences are probably related to the fact that both in Beckley et al. (2007) and our investigation the geographical latitudes covered by the real orbit inclination are not the same with the simulated case scenario analyzed by Morel and Willis (2005).

Fig. 6 illustrates the observed geographical MSL trend resulting from the geocenter motion model applied in the SLR/DORIS stations from Cheng et al. (2010) over Jason-2 cycles 001 to 074 (2 years). For the whole period of the SLR/DORIS orbits, the negligence of just the annual term of the geocenter motion correction creates an apparent MSL rise of $0.14 \pm 0.35 \mathrm{~mm} / \mathrm{yr}$ in 2 years. This is a very important result because if orbits based on SLR/DORIS and GPS are used during the inter-mission calibration phases of TOPEX/Poseidon, Jason-1, Jason-2 and future Jason-3 then the omission of the geocenter motion correction could potentially affect the inter-mission calibration of the altimeter data.

Finally, by editing the SLR weighted residuals after the application of the geocenter motion correction we see a slight improvement with the annual term of the Cheng et al. (2010) model over not using any geocenter motion in the SLR/DORIS orbits. For the gpsdyn orbits the residuals remain unchanged.

All the above, in addition to the small improvement in the SLR weighted residuals, demonstrate that the geocenter motion correction should be included as a standard in the Jason-2 POD process.

\section{Conclusions}

In conclusion, we have characterized the spurious signals contained in the origin of the Jason-2 orbits from an analysis of GSFC's SLR/DORIS-based and GPS-based dynamic and a set of reduced-dynamic orbits. A 118-day dominant signal was found in the $\mathrm{X}$ and Y components of the comparison gpsdyn to the SLR/DORIS orbits. The 118-day signal is the precise draconitic (beta-prime) period for the Jason satellites and it is due to solar radiation pressure (SRP) mis-modeling. This result is consistent to the analysis by Zelensky et al. (2010). In the Z-component the annual signature has the largest amplitude. Other signals of lower amplitude appear at the 87-days and 112-days close to the 4th and 3rd harmonics of the GPS draconitic year of 351 days (Schmid et al. 2007), but their origin still remains unknown. We've shown that the comparison of the gpsdyn to the 
SLR/DORIS orbits exhibits a large annual signal in the Z-component suggesting a motion of the origin between the two orbit sets. The jpll1a orbits (Bertiger et al. 2010) also exhibit an annual signature in $\mathrm{Z}$ when compared to the gpsdyn orbits but of smaller amplitude. This left an open question for further investigation with respect to the consistency in the force modeling and the origin of the two analysis centers GPS orbit sets.

We examined the centering of the SLR/DORIS orbits with respect to the gpsdyn orbits after the introduction of the annual geocenter motion as a degree-1 loading displacement correction in the stations. For the geocenter motion correction we have used two models from Cheng et al. (2010) and Swenson et al. (2010). After the introduction of the annual geocenter motion correction from Swenson et al. (2010) and Cheng et al. (2010) in the POD process of the $S L R / D O R I S$ orbits, the initial translational variations of the origin with respect the gpsdyn orbits, dropped down from $2.82 \mathrm{~mm}$ in $2.10 \mathrm{~mm}$ and $1.17 \mathrm{~mm}$ respectively. Furthermore, the 7-parameter transformation between the gpsred and gpsdyn orbits, demonstrated that both orbits sets have a very consistent Z-origin. Based on these facts, we have concluded that our gpsdyn orbits closely follow the CM consistent with our conservative force modeling, while the SLR/DORIS are centered closer to the origin of the ITRF, which is the CF for sub-secular scales. Moreover, our investigation suggests that any SRP mis-modeling error on Jason-2 is not responsible for the annual signature seen in the Z-component comparison between jpll1a and the GSFC's gpsdyn orbits. This fact left an open question of whether this annual signal is due to some difference in the POD modeling, such as the time variable gravity, or whether is due to an origin motion as is proven to be the case with GSFC's $S L R / D O R I S$ orbits.

We have characterized the errors that would be seen by the users of satellite altimetry data when using the SLR/DORIS std0905-based Jason-2 orbits without a priori knowledge of the geocenter motion. The SLR/DORIS orbits, in which the annuals term of the geocenter motion has been taken in to account, exhibited a noticeable annual effect in the Z-component of the orbit frame of $74 \%$ and $81 \%$ compared to each geocenter model. In the case of the gpsdyn orbits this effect was found to be only $16 \%$ and $19 \%$ respectively with insignificant amplitude. The gpsdyn orbits remained practically insensitive to the applied geocenter motion correction. We have depicted that in the case of the $S L R / D O R I S$ orbits the geographical amplitude of the mean radial orbit error (DH) is significantly larger and can reach $2.5 \mathrm{~mm}$ in the poles. Moreover, our transfer function that connects the error in the Z-component of the Jason-2 orbit frame, from the omission of the annual geocenter motion correction, to the MSL error of both the gpsdyn and $S L R / D O R I S$ orbits is closer to the transfer function re-calculated from the study of Beckley et al. (2007) and slightly differs from the one from Morel and Willis (2005). It is worth noticing that it is the first time where the study of Morel and Willis (2005) has been revisited with real case scenarios

In this study we addressed only the annual term of the modeled geocenter motion as a degree-1 loading displacement to the tracking stations that participate in the POD process. Indeed, seasonal geocenter motion results from mass transfer at the Earth's surface. We do model a big part of the degree-1 signal, but this is only a portion of the 
degree-1 deformation since we also have associated deformation. The tracking network displaces also because of higher degrees. Future work could focus on the forward modeling of the seasonal displacements at the stations together with the complete geocenter model correction.

We have found that the omission of just the geocenter annual term can contribute to an apparent $0.14 \mathrm{~mm} / \mathrm{yr}$ error in the MSL estimates in 2 years based on the std0905 SLR/DORIS orbits. However, although the annual term of the geocenter motion could reflect a stationary signal in time, the whole geocenter motion is a non-stationary process that includes secular trends. For example global ice melting on the Earth has been found to induce long-term displacements of the geocenter particularly along the Z-axis, toward the North Pole. Métivier et al. (2010) have calculated that the geocenter velocity can reach $0.7-0.8 \mathrm{~mm} / \mathrm{yr}$ and is today most probably between 0.3 and $0.8 \mathrm{~mm} / \mathrm{yr}$. Especially in the last decade it seems that there's an increase in the geocenter velocity not superior to $0.5 \mathrm{~mm} / \mathrm{yr}$. Since one of the main objectives in the present development of altimetry MSL is stability at the $0.1 \mathrm{~mm} / \mathrm{yr}$ level (Cazenave et al. 2009), it would be very interesting to extend the current study to the whole period of Jason-1 and Jason-2 with a complete geocenter motion correction. Our results have shown that the Jason-2 GPS and $S L R / D O R I S$ orbits respond differently to the omission of an annual geocenter model in the POD process. Hence, if orbits based on SLR/DORIS and GPS are used during the intermission calibration phases (e.g. TOPEX vs. Jason-1; Jason-1 vs. Jason-2), then the geocenter model omission error could potentially affect the intermission calibration of altimeter data. We need to elucidate whether this conclusion still applies if other techniques are used to process GPS data than the ones we have applied in this paper using fixed and filtered IGS orbits, and GPS double differences on Jason-2.

\section{Acknowledgements}

This research was supported by the following NASA Programs: NNH09ZDA001N-IDS: Interdisciplinary Research in Earth Science (IDS) and NNH07ZDA001N-OSTST: Ocean Surface Topography Science Team. We thank the two anonymous reviewers, Dr. Xavier Collilieux and the editor Dr. Pascal Willis for their constructive comments on this manuscript.

\section{References}

Altamimi, Z., X. Collilieux, J. Le Grand, B. Garyt, and C. Boucher, 2007. ITRF2005: A new release of the International Terrestrial Reference Frame based on time series of station positions and Earth orientations parameters, J. Geophys. Res., doi:10.1029/2007JB004949, in press.

Beckley B D, F G Lemoine, S B Luthcke, R D Ray and N P Zelensky, 2007. A reassessment of global and regional mean sea level trends from TOPEX and Jason-1 altimetry based on revised reference frame and orbits, Geophys. Res. Lett. 34, L14608, doi:10.1029/2007GL030002 
Bertiger, W. I., Bar-sever Y E, Christensen E J, et al. 1994. GPS precise tracking of TOPEX/POSEIDON: Results and implications, J. Geophys. Res., 99(C12), 24,44924,464, doi:10.1029/94JC01171.

Bertiger W, Shailen D D, Dorsey A, Haines J B, Harvey N, Kuang D, Sibthorpe A, Weiss P J, 2010. Sub-Centimeter Precision Orbit Determination with GPS for Ocean Altimetry, Mar. Geod. 33(S1):363-378, doi:10.1080/01490419.2010.487800

Blewitt, G., 2003. Self-consistency in reference frames, geocenter definition, and surface loading of the solid Earth. J. Geophys. Res. 108 (B2), doi:10.1029/2002JB002290, Art. No. 2103.

Blewitt, G., D. Lavallée, P. Clarke, K. Nurutdinov, 2001, A New Global Mode of Earth Deformation: Seasonal Cycle Detected, Science vol 294, pp 2342-2345

Boehm J, Niell A, tregoning P, Schuh H, 2006. Global Mapping Function (GMF): a new empirical mapping function based on numerical weather model data. Geophys res Lett 33:L07304. doi:10.1029/2005GL025546

Boehm J, Heinkelmann R, Schuh H, 2007. Short note : a global model of pressure and temperature for geodetic applications. J Geod 81(10):679-683, doi:10.1007/s00190007-0135-3

Cazenave, A. D.P. Chambers, P. Cipollini, L.L. Fu, J.W. Hurrell, M. Merrifield, S. Nerem, H.P. Plag, C.K. Shum, Josh Willis, 2010. "Sea Level Rise - Regional and Global Trends" in Proceedings of OceanObs'09: Sustained Ocean Observations and Information for Society (Vol. 1), Venice, Italy, 21-25 September 2009, Hall, J., Harrison, D.E. \& Stammer, D., Eds., ESA Publication WPP-306, doi:10.5270/OceanObs09.pp.11

Cerri L, JP Berthias, WI Bertiger, BJ Haines, FG Lemoine, F Mercier, JC Ries, P. Willis, NP Zelensky, M Ziebart (2010), Precision Orbit Determination Standards for the Jason Series of Altimeter Missions, Mar Geodesy, 33(S1):379-418, doi:10.1080/01490419.2010.488966

Chen, J.L., Wilson, C.R., Eanes, R.J., Nerem, R.S., 1999. Geophysical interpretation of observed geocenter variations. J. Geophys. Res. 104 (B2), 2683-2690.

Cheng M, J Ries, B Tapley, 2010. Geocenter Variations From Analysis of SLR data, IAG Symp., 138, inpress

Collilieux, X., Altamimi, Z., Ray, J., van Dam, T., Wu, X., 2009. Effect of the satellite laser ranging network distribution on geocenter motion estimation. J. Geophys. Res. 114, Art. No. B04402.

Colombo O.L, 1986. Ephemeris errors of GPS satellites. Bull Geod, 60, 64-84

Dong, D., Yunck, T., Heflin, M., 2003. Origin of the international terrestrial reference frame. J. Geophys. Res. 108 (B4), Art. No. 2200.

Ferland R, Bourassa M, 2006. From Relative to Absolute Phase Center Calibration: The effect on the SINEX Products, presented at the International GNSS workshop "NearEarth Navigation \& Geodesy at ESOC, European Space Agency, Darmstadt, Germany. Available from <http://nng.esoc.esa.de/ws2006/session10.html>

Fritsche M, R Dietrich, A Rulke, M Rothacher, P Steigenberger, 2010. Low-degree earth deformation from reprocessed GPS observations, GPS Solut (2010) 14:165-175, doi: 10.1007/s10291-009-0130-7

Hedin A E, 1987. MSIS-86 thermospheric model. J. Geophys. Res. 92(A5), pp 46494662. 
Kang, Z.G., Tapley, B., Chen, J.L., Ries, J., Bettadpur, S., 2009. Geocenter variations

Klinker E, Rabier F, Kelly G, Mahfouf J F, 2000. The ECMWF operational implementation of four-dimensional variational assimilation. Part III : experimental results and diagnostics with operational configuration. Q. J. R. Meteorol. Soc. 126, 1191

Knocke P C, Ries J C, Tapley B D, 1988. Earth radiation pressure effects on satellites in: Proceedings of the AIAA/AAS Astrodynamics Conference, Minneapolis, Minnesota, pp. 577-586, August 15-17.

Lavallée, D. A., T. van Dam, G. Blewitt, and P. J. Clarke (2006), Geocenter motions from GPS: A unified observation model, J. Geophys. Res., 111, B05405, doi:10.1029/2005JB003784

Lemoine F G, N P Zelensky, D S Chinn, D E Pavlis, D D Rowlands, B D Beckley, S B Luthcke, P Willis, M Ziebart, A Sibthorpe, J P Boy, V Luceri, 2010. Towards development of a consistent orbit series for TOPEX, Jason-1, and Jason-2, Adv in Space Res, doi:10.1016/j.asr.2010.05.007

Lemoine J M, Bruinsma S, Loyer S, et al, 2007. Temporal gravity field models inferred from GRACE data. Adv Space Res 39 (10), 1620-1629, doi:10.1016/j.asr.2007.03.062

Luceri V, Bianco G, 2007. The temporary ILRS reference frame : SLRF2005, International Laser Ranging Service Fall Meeting, 24-28 September 2007, Grasse, France. Available from http://www.oca.eu/gemini/ecoles_colloq/colloques/ilrs2007/articles.php?lng=enandp $\mathrm{g}=98$.

Luthcke SB, NP Zelensky, DD Rowlands, FG Lemoine, TA Williams, 2003. The 1Centimeter Orbit: Jason-1 Precision Orbit Determination Using GPS, SLR, DORIS, and Altimeter Data, Mar Geodesy, 26: 399-421, doi:10.1080/01490410390256727

Luthcke, S.B., Rowlands, D.D., Lemoine, F.G., et al., 2006. Monthly spherical harmonic gravity field solutions determined from GRACE intersatellite range-rate data alone. Geophys. Res. Lett 33 (2), L02402, doi:10.1029/2005G1024846

McCarthy, D. D., G. Petit (Eds.), IERS Conventions (2003), IERS Tech. Note 32, Verlag des Bundesamts fur Kartographie und Geodasie, Frankfurt am Main. 2004.

Métivier L, Greff-Lefftz M and Altamimi Z, 2010. On secular geocenter motion: The impact of climate changes, Earth and Planet. Sc. Lett., 296, pp. 360-366, doi:10.1016/j.eps1.2010.05.021

Melachroinos S A, F G Lemoine, N P Zelensky, D D Rowlands, C Deng, D E Pavlis, S B Luthcke, S M Klosko, 2011a. Status of Precise Orbit Determination for Jason-2 using the GPS, SLR, \& DORIS data at NASA/GSFC, paper presented at the European Geosciences Union General Assembly, EGU2011-10409, Vienna, Austria, Available at < presentations.copernicus.org/EGU2011-2697_presentation.pdf>

Melachroinos S A, F G Lemoine, N P Zelensky, D D Rowlands, S B Luthcke, D S Chinn, S M Klosko, J Dimarzio, D E Pavlis, O Bordyugov, 2011b. Jason-2 systematic error analysis in the GPS derived orbits, paper presented at the American Geosciences Union General Assembly, G41B-0740, San Francisco, CA, USA. Available at < http://agu-fm11.abstractcentral.com> 
589

590

591

592

593

594

595

596

597

598

599

600

601

602

603

604

605

606

607

608

609

610

611

612

613

614

615

616

617

618

619

620

621

622

623

624

625

626

627

628

629

630

631

632

633

634
Morel L and P Willis (2005), Terrestrial reference frame effects on global sea level rise determination from TOPEX/Poseidon altimetric data, Adv in Space Res, doi:10.1016/j/asr.2005.05.113

Pavlis D E, Deng C, Poulose S et al., GEODYN Operations Manuals, Contractor Report, SGT Inc., Greenbelt, Maryland, 2009

Pearlman M R, Degnan J J, Bosworth J M, 2002. The International Laser Ranging Service, Adv Space Res 30 (2), 135-143,doi:10.1016/S0273-1177(02)00277-6

Ray J, Altamimi Z, Collilieux X, van Dam T, 2008. Anomalous harmonics in the spectra of GPS position estimates. GPS solute. 12, 55-64, doi:10.1007/s10291-007-0067-7

Ray R D, 1999. A global ocean tide model from TOPEX/Poseidon altimetry: GOT99.2, NASA TM-1999-209478, NASA Goddard Space Flight Center

Ries J C, LPOD2005: a practical realization of ITRF2005 for SLR-based POD in: Presentation, OSTST, Nice, France, November 2008. Available at <http://www.aviso.oceanobs.com/fileadmin/documents/OSTST/2008/oral/ries.pdf>

Schmid R, Steigenbeger P, Gendt G, Ge M, Rothacher M, 2007. Generation of a consistent absolute phase center correction model for GPS receiver and satellite antennas, J. Geod. 81 (12), 781-798, doi:10.1007/s00190-007-0148-y

Swenson S, D Chambers, J Wahr (2008), Estimating geocenter variations from a combination of GRACE and ocean model output, JGR, vol. 113, B084410, doi:10.1029/2007JB005338

Tavernier G, Fagard H, Feissel-Vernier M, 2006. The International DORIS Service: genesis and early achievements. J. Geod. 80 (8-11), 403-417, doi:10.1007/s00190006-0082-4

Urschl C, Beutler G, Gurtner W, et al. 2007, Contribution of SLR tracking data to GNSS orbit determination, Adv in Space Res., Vol. 39, Issue 10, p. 1515-1523 doi: 10.1016/j.asr.2007.01.038

Willis P, Ries J C, Zelensky N P, Soudarin L, Fagard H, Pavlis E C, Lemoine F G, 2009. DPOD2005 : An extension of ITRF2005 for Precise Orbit Determination, Adv. Space Res., 44(5), 535-544, DOI: 10.1016/j.asr.2009.04.018

Willis P, Fagard H, Ferrage P, 2010. The International DORIS Service (IDS): toward maturity. Adv. Space Res. 45 (12), 1408-1420, doi:10.1016/j.asr.2009.11.018

Wu X, J Ray, T van Dam, 2012. Geocenter motion and its geodetic and geophysical implications, J. Geodyn. 58, 44-61, doi:10.1016/j.jog.2012.01.007

Zelensky NP , FG Lemoine, M Ziebart, A Sibthorpe, P Willis, BD Beckley, SM Klosko, DS Chinn, DD Rowlands, SB Luthcke, DE Pavlis, V Luceri, 2010. DORIS/SLR POD modelling improvements for Jason-1 and Jason-2, Adv. in Space Res., Vol. 46, Issue 12, p. 1541-1558, doi:10.1016/j.asr.2010.05.008 
635 Table 1: GSFC Jason-2 GPS POD model standards: std0905

\section{Reference Frame and displacement of reference points}

\begin{tabular}{|l|l|}
\hline GPS & 38 IGS05 (Ferland and Bourassa 2006) TRF stations \\
\hline $\begin{array}{l}\text { Tidal CoM and } \\
\text { EOP }\end{array}$ & $\begin{array}{l}\text { GOT4.7 (update to Ray, 1999); VLBI high frequency } \\
\text { terms }\end{array}$ \\
\hline Ocean Loading & GOT4.7 (update to Ray, 1999) all stations \\
\hline Earth tide & IERS2003 \\
\hline EOP & $\begin{array}{l}\text { IERS Bulletin A daily consistent with ITRF2005 } \\
\text { (Altamimi et al. 2007) }\end{array}$ \\
\hline $\begin{array}{l}\text { Precession } \\
\text { Nutation }\end{array}$ & IAU2000 \\
\hline
\end{tabular}

Satellite surface forces and attitude

\begin{tabular}{|l|l|}
\hline Albedo/IR & Knocke-Ries-Tapley (1988) \\
\hline Atmospheric drag & MSIS86 (Hedin, 1987) \\
\hline Radiation pressure & 8-panel, CR=0.916 (tuned) \\
\hline Attitude & Quaternions \\
\hline Tracking data and parameterization \\
\hline Tracking data & $\begin{array}{l}\text { Double Difference LC iono-free tracking data, float } \\
\text { ambiguities, fixed and filtered IGS sp3 orbits }\end{array}$ \\
\hline $\begin{array}{l}\text { Troposphere } \\
\text { modeling }\end{array}$ & $\begin{array}{l}\text { 1/hr scale(wet+dry) troposphere (GMF (Boehm et al. } \\
\text { 2006)/GPT (Boehm et al. 2007)-Hopfield) } \\
\text { adjusted using 2 paths (1 station + 2 GPS s/c) during the } \\
\text { POD }\end{array}$ \\
\hline Parameterization & $\begin{array}{l}\text { arc }: \text { 24+6 h long } \\
\text { gpsdyn }: \text { drag 1/8hr, 2 OPR along\&cross / arc } \\
\text { gpsred }: \text { drag 1/arc, OPR along\&cross/ 15 min, sigma } \\
=1 . \mathrm{e}-09, \text { correl time =3600 sec }\end{array}$ \\
\hline Antenna Reference & PCOs and PCVs : igs05.atx \\
\hline GPS stations + &
\end{tabular}


639

640

641

642

643

644

645

646

647

648

649 Table 2: Description of the Jason-2 orbit solutions used in this study

\begin{tabular}{l|l}
\hline POD name used in the text & \multicolumn{1}{l}{ Description } \\
\hline gpsdyn & GSFC's GPS dynamic \\
gpsred & GSFC's GPS reduced dynamic \\
gpsdyn_com_csr_ & GSFC's GPS dynamic + Cheng et al. 2010 \\
gpsdyn_com_swn_- & CoM correction \\
SLR/DORIS & GSFC's GPS dynamic + Swenson et al. 2008 \\
CoM correction
\end{tabular}

JPL GPS antenna PCV map consistent with igs05.atx, 636 Revised LC GPS antenna PCO offsets 
661 Table 3: Z-component annual amplitudes ( $\mathrm{mm})$ from each geocenter motion model and 662 orbit transformations compared to the ratios of reduction in the annual signature to each 663 geocenter motion model and the SLR/DORIS - gpsdyn comparison.

\begin{tabular}{|c|c|c|c|c|}
\hline Geocenter model & $\begin{array}{c}\text { Annual } \\
\text { Amplitude }\end{array}$ & \multirow{5}{*}{$\begin{array}{l}\text { Geocenter } \\
\text { model } \\
\text { applied }\end{array}$} & \multirow{5}{*}{$\begin{array}{l}\text { Ratio of the } \\
\text { reduction to } \\
\text { the } \\
\text { SLR/DORIS } \\
\text { - gpsdyn } \\
\text { signal }\end{array}$} & \multirow{5}{*}{$\begin{array}{l}\text { Ratio of } \\
\text { the } \\
\text { reduction } \\
\text { to each } \\
\text { model }\end{array}$} \\
\hline Swenson et al. 2010 & 1.85 & & & \\
\hline Cheng et al. 2010 & 4.24 & & & \\
\hline $\begin{array}{l}\text { Helmert } \\
\text { transformation (ref. } \\
\text { orbit } g p s d y n \text { ) }\end{array}$ & $\begin{array}{c}\text { Annual } \\
\text { Amplitude }\end{array}$ & & & \\
\hline SLR/DORIS & 2.82 & & & \\
\hline SLR/DORIS_com_swn & 2.10 & $\begin{array}{l}\text { Swenson et } \\
\text { al. } 2010\end{array}$ & $25 \%$ & $39 \%$ \\
\hline SLR/DORIS_com_csr & 1.17 & $\begin{array}{c}\text { Cheng et al. } \\
2010\end{array}$ & $58 \%$ & $39 \%$ \\
\hline
\end{tabular}

664

665

666 667

668

Table 4: Z-component annual amplitudes (mm) and ratios from each orbit solution after the geocenter motion correction to each model

\begin{tabular}{|l|c|c|c|c|}
\hline Helmert transformation & Ref. Orbit & $\begin{array}{c}\text { Amplitude } \\
(\mathrm{mm})\end{array}$ & $\begin{array}{c}\text { Ratio of } \\
\text { Phase } \\
\text { the resulted } \\
\text { signature } \\
\text { to each } \\
\text { model }\end{array}$ \\
\hline gpsdyn_com_swn & gpsdyn & 0.3 & 62.7 & $16 \%$ \\
\hline gpsdyn_com_csr & gpsdyn & 0.8 & 4.9 & $19 \%$ \\
\hline SLR/DORIS_com_csr & SLR/DORIS & 3.1 & 7.2 & $74 \%$ \\
\hline SLR/DORIS_com_swn & SLR/DORIS & 1.5 & 64.3 & $81 \%$ \\
\hline
\end{tabular}

669 Table 5: Effect observed on the derived mean sea level (DH) resulting from the Cheng et 670 al. (2010) geocenter motion correction in the gpsdyn and SLR/DORIS stations for Jason-2 671 cycle 058 (Jan 28-Feb 07, 2010)

\begin{tabular}{|c|c|c|c|c|}
\hline Orbit comparisons & Ref. Orbit & $\mathrm{Tz}(\mathrm{mm})$ & $\mathrm{DH}(\mathrm{mm})$ & $\mathrm{DH} / \mathrm{Tz}$ \\
\hline SLR/DORIS_com_csr & SLR/DORIS & $-4.67 \pm 3.40$ & $1.06 \pm 2.66$ & -0.22 \\
\hline gpsdyn_com_csr & gpsdyn & $-0.82 \pm 0.28$ & $0.17 \pm 0.37$ & -0.21 \\
\hline
\end{tabular}



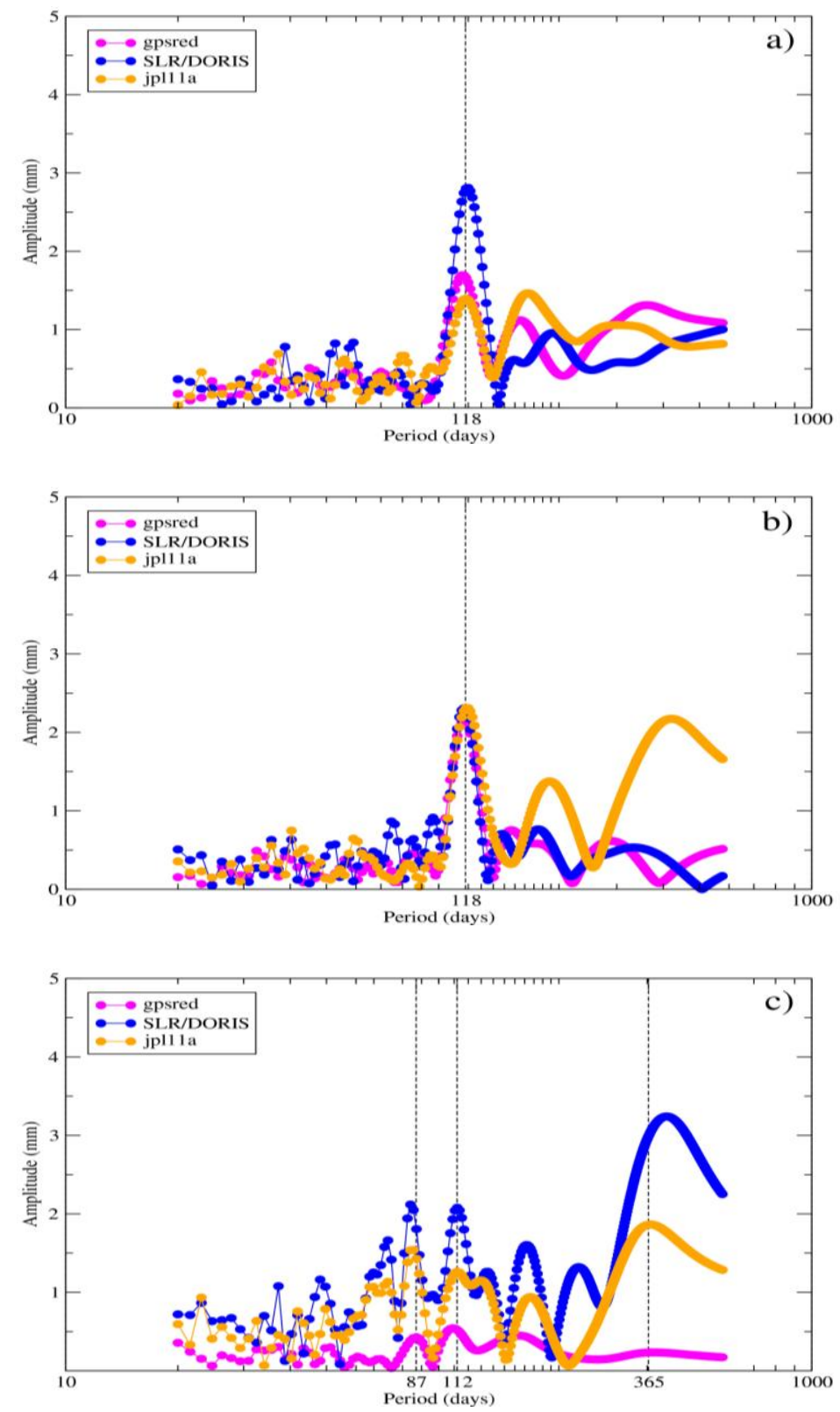

677 Figure 1: Periodogram (in $\mathrm{mm}$ ) of the orbit origins after a 7-parameter Helmert 678 transformation between the NASA GSFC Jason-2 GPS-based dynamic orbits and the 679 three test orbits: NASA GSFC Jason-2 GPS-based reduced-dynamic (gpsred), NASA 680 GSFC Jason-2 SLR/DORIS dynamic and JPL Jason-2 GPS-based reduced-dynamic 681 (jpll la). a) X-component, b) Y-component, c) Z-component. In purple, blue and orange 682 are the comparisons to GSFC's gpsred, SLR/DORIS dynamic orbits and jpll1a GPS683 based reduced dynamic orbits respectively. 684 

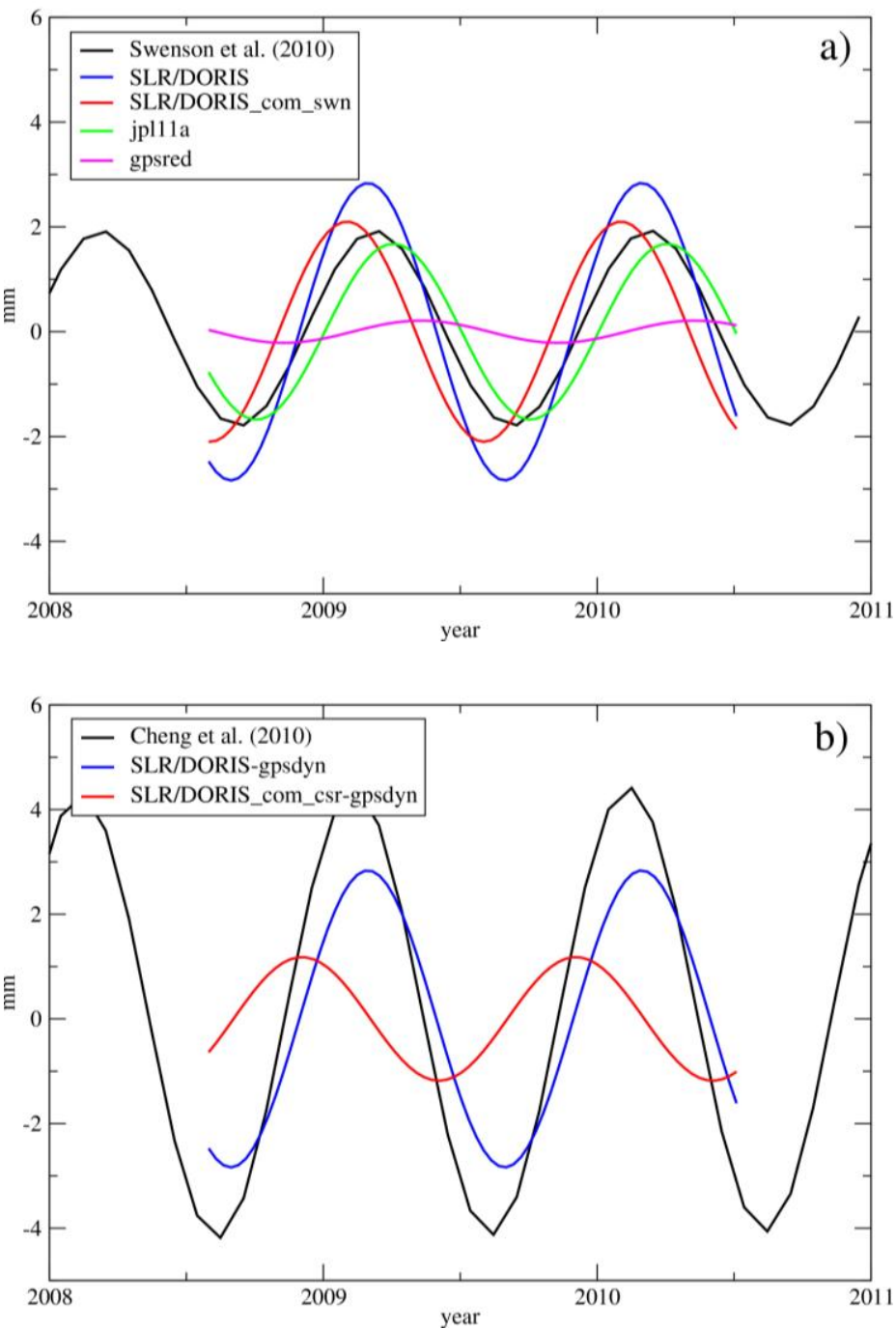

686

687 Figure 2: Jason-2 fitted annual signal of the Z-component time-series from the 7688 parameter transformation between the gpsdyn (reference orbit) and the test orbits: $689 S L R / D O R I S$, jpll1a and gpsred. a) compared to the Swenson et al. (2010) applied only in 690 the SLR/DORIS orbits, b) compared to the Cheng et al. (2010) applied only to the $691 \quad S L R / D O R I S$ orbits 692 693 694 695 696 697 

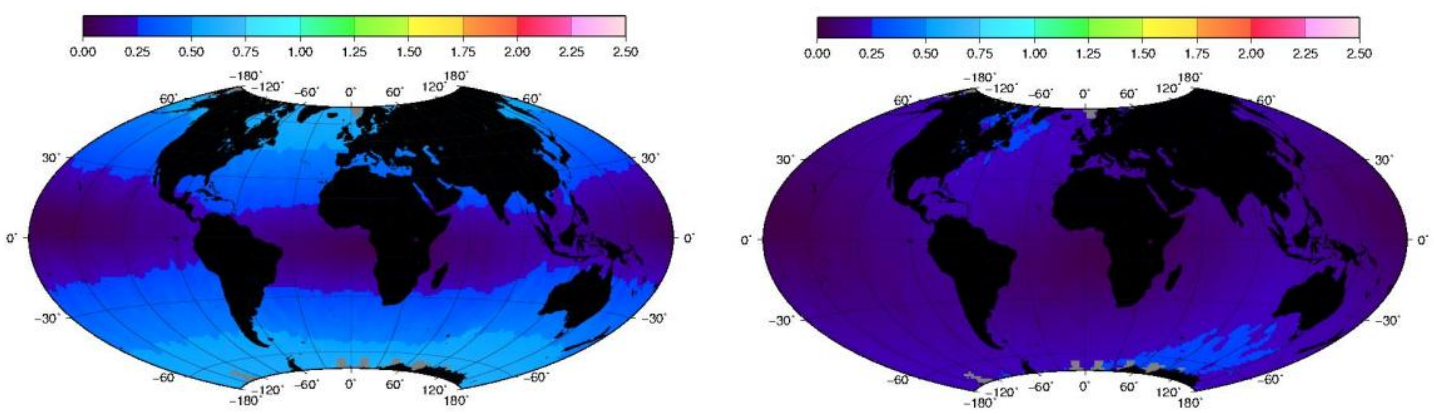

699
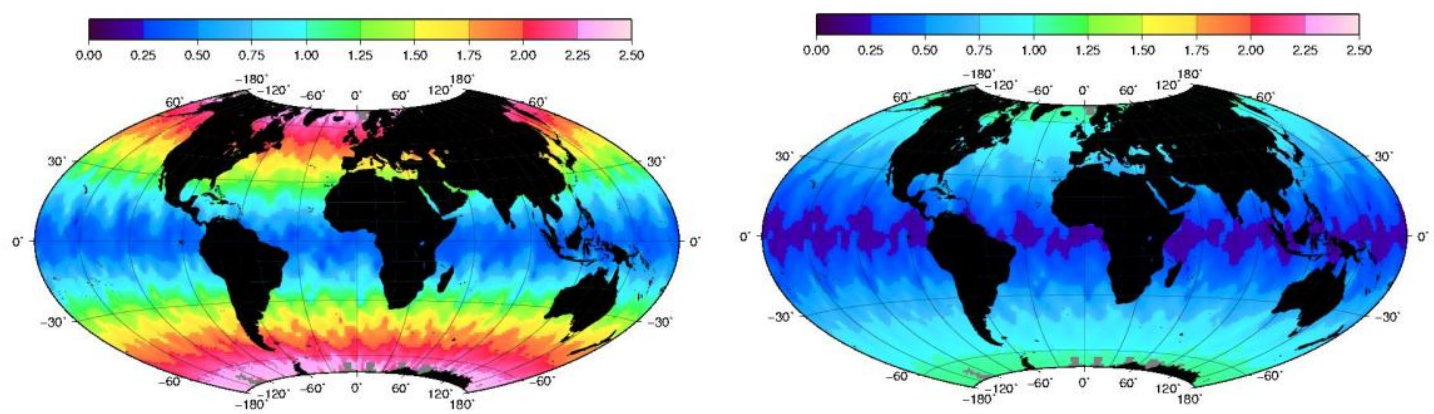

700 Figure 3: Amplitude (in $\mathrm{mm}$ ) of the geocenter motion correction as it maps into the radial 701 orbit differences (DH) of the gpsdyn (up) and the SLR/DORIS (bottom) orbit frame. Left 702 from Cheng et al. (2010) and right from Swenson et al. (2010).

703
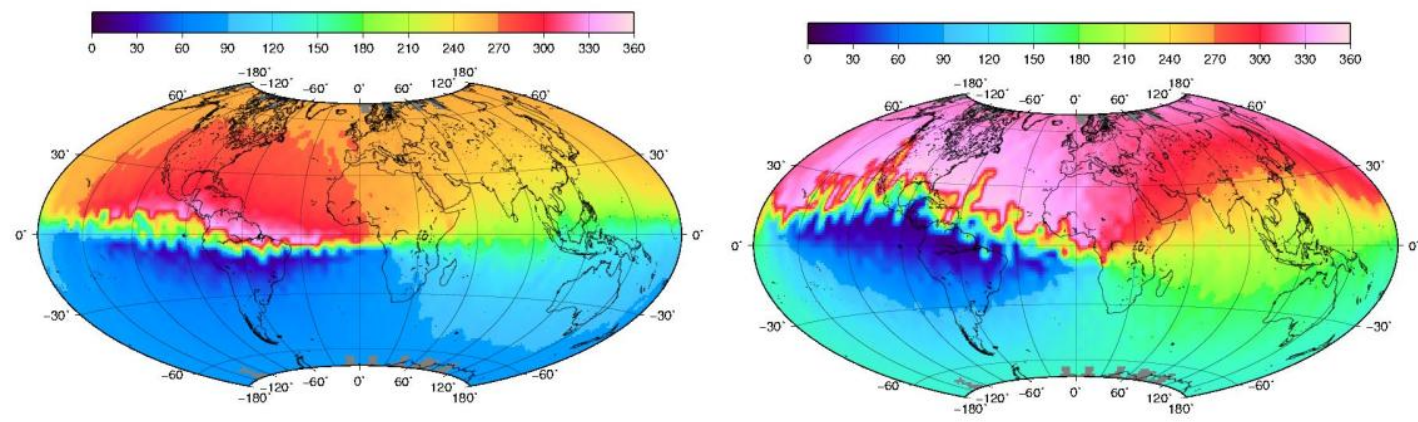

704
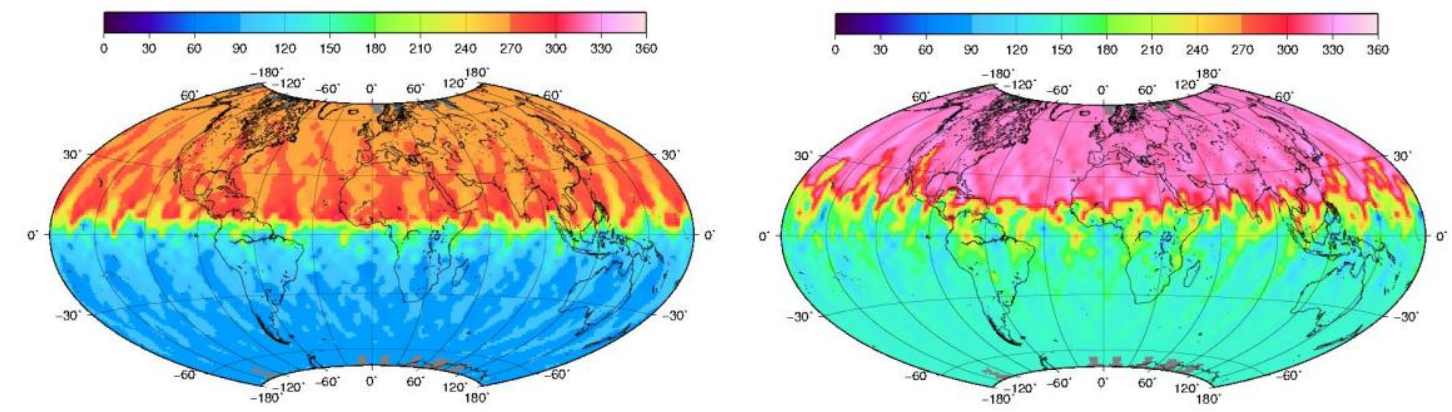

705

Figure 4: Phase (in degrees) of the geocenter motion correction as it maps into the radial 706 orbit differences (DH) of the gpsdyn (up) and the SLR/DORIS (bottom) orbit frame. Left 707 from Cheng et al. (2010) and right from Swenson et al. (2010). 
709

711 Figure 5: Observed geographical MSL error (in $\mathrm{mm}$ ) resulting from the geocenter motion 712 model of the SLR/DORIS stations from Cheng et al. (2010) for Jason-2 cycle 058 (Jan 28-

713 Feb 07, 2010)

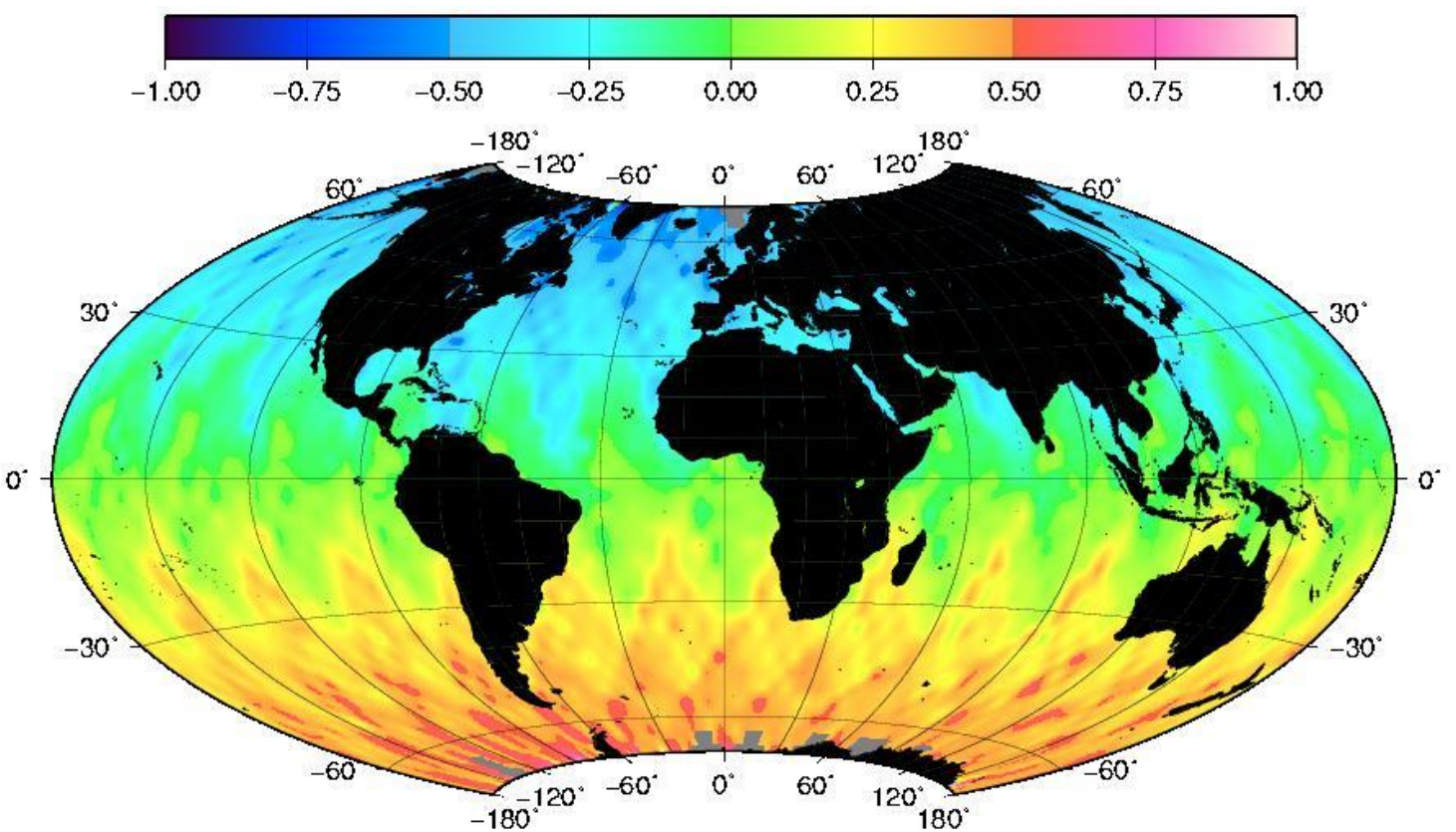

714

715

716

Figure 6: Observed geographical MSL trend (in $\mathrm{mm} / \mathrm{yr}$ ) resulting from the geocenter motion model of the SLR/DORIS stations from Cheng et al. (2010) over Jason-2 cycles 717 001 to 074 ( 2 years) : $0.14 \pm 0.35 \mathrm{~mm} / \mathrm{yr}$
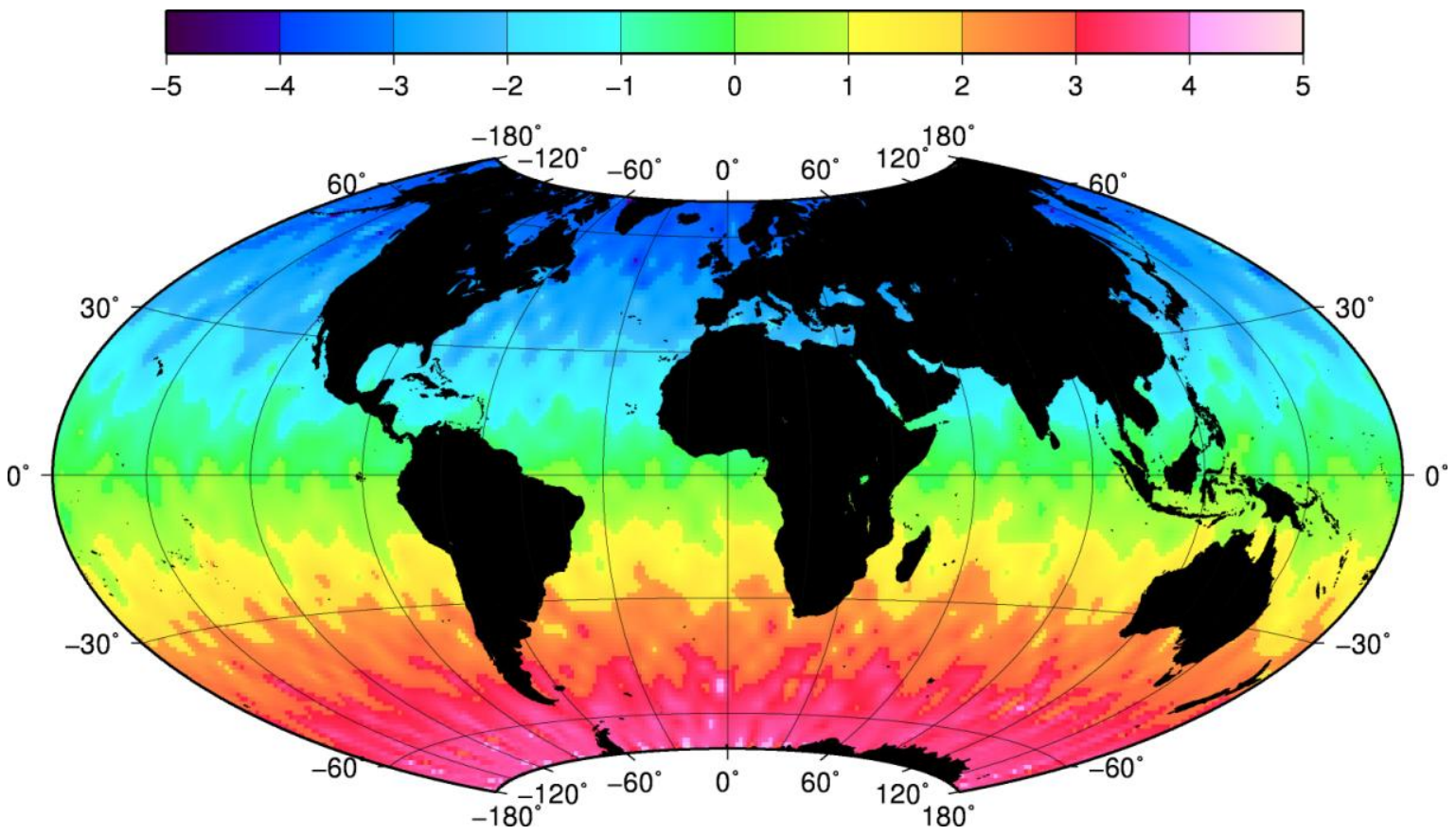

718 
Rev. 1

While it appears necessary, as demonstrated in this paper, that geocenter motion should be applied in the POD process, so that all techniques provide orbits that are internally consistent, the authors may wish to consider (or pose to the altimetry community), whether the resulting orbits (presumably now properly centered at the $\mathrm{CM}$ ) should in fact then be translated to the CF frame for purposes of computing sea level. The question arises since sea level is generally measured relative to the crust (e.g., tide gauges). Stated alternatively, should orbits used for sea level analysis really be in the CM frame or should they translated to the CF frame? This question would apply to the non-tidal as well as shorter period tidal geocenter motion.

Indeed, the reviewer is posing a very interesting and challenging question concerning modern and current geodetic sea-level investigations. Our future investigation is concentrating at exactly trying to answer to this question with 2 abstracts and one proposal recently submitted.

A tide gauge directly measures the displacement of the sea surface relative to a land point, and so would seem ideal. However the use of tide-gauge data alone to infer global measures of sea-level change is fundamentally problematic due to processes that intervene in the relative motions of the sea surface and the solid Earth on which the tide gauges are, over a broad range of spatial and temporal scales. The land beneath the tide-gauges is subject to motions such GIA (secular trends), coastal erosion, sedimentary loading, subsidence, atmospheric loading, tectonic processes and the different ocean/land response to present-day mass redistributions such as cryospheric loading and terrestrial hydrological loading. Another point is that the land motion near the costs on which the tide gauge are, provides a very poor sample distribution of the Earth deformation processes that will generally not average out on the global scale. Secondly, the vertical land motion from geodetic techniques used for the correction of the relative sea-level trends from the tide gauge sites, are subject to terrestrial reference frame errors. Also, an error in the terrestrial reference frame origin at the center of mass of the Earth implies an error in the height of sea surface inferred by satellite altimetry observations. Orbits whose origin is closer centered at the center of Mass of the Earth system, would ideally be insensitive to those reference frame realization errors. Tide gauges are immune to terrestrial reference frame errors only if the vertical velocities used for the correction of the relative sea level rates are inferred by other technique (cf. internal rates by G. Mitchum studies) and thus can be used only locally as the ground truth of calibration/validation for the satellite altimetry. In order though for these two types of sea-level observations to be comparable they need to be defined in the same ref. frame origin. For the tide gauges the origin is the center of the Solid Earth (CE) where as those of the satellite altimetry "ideally" is the center of mass of the Earth system (CM) defined by the satellite orbit dynamics. However the CE is never a frame of reference that can be realized by space geodesy. In GPS practice, for example, the CF frame is most commonly used. Also we must not forget that in order for the two types of 
sea-level observations to be comparable, the vertical motions (from GPS heights normal to a geocentric ellipsoid) must be removed from the tide gauges records. So the way this vertical motion is defined is very important for the intercomparisons, which means improved reference frames that for example would take into account seasonal mass redistribution, its effect on degree-1 deformation and therefore its effect on the frame origin, are needed.

Rev. 2

All suggested changes have been applied. 